\title{
A parametric data handling evaluation framework for autonomous lunar networks
}

\author{
Jasmine Rimani ${ }^{1,2} \cdot$ Luigi Mascolo $^{1}$ (1) Juan A. Fraire ${ }^{3,4,5}$
}

Received: 30 November 2020 / Revised: 23 July 2021 / Accepted: 7 September 2021 / Published online: 22 September 2021

(c) The Author(s) 2021

\begin{abstract}
Different and exciting exploration opportunities toward the Moon are opening in this decade. In particular, the major space agencies are putting a considerable effort in designing and studying a broad spectrum of missions that will bring back the humans on the Moon. During the evaluation of Lunar mission concepts, having a tool that can quickly assess the best communication and data-handling architecture given a set of satellites and a site of interest is mandatory. In this work, a novel parametric framework is presented and applied to the study of the expected connectivity of Lunar networks. The framework comprises bent-pipe, store-and-forward and store-carry-and-forward networking approaches, covering most common data management options. The methodology is designed to determine the best communication architecture given an arbitrary set of available satellites, ground stations, point of interest, and data volume. The proposed algorithm has been applied in a motivating case study of a networked mission devoted to observing lava tubes sites on the Moon surface. Results validate the approach which can identify the inflection points where different data handling techniques outperform each other.
\end{abstract}

Keywords Communication $\cdot$ Moon $\cdot$ Lava tubes $\cdot$ Space networking $\cdot$ Access analysis

\section{Introduction}

Many space agencies, including NASA and ESA, foresee that mankind will be back on the Moon in the 2020s [1]. In this context, companies and universities are engaged in the definition of a wide spectrum of different mission architectures and concepts $[2,3]$. At the same time, the general

Luigi Mascolo

luigi.mascolo@polito.it

Jasmine Rimani

jasmine.rimani@polito.it ; jasmine.rimani@isae-supaero.fr

Juan A. Fraire

juanfraire@gmail.com

1 Department of Mechanical and Aerospace Engineering, Politecnico di Torino, Torino, Italy

2 Department of Aerospace Vehicles Design and Control (DCAS), ISAE-SUPAERO, Toulouse, France

3 CONICET-Universidad Nacional de Córdoba, Córdoba, Argentina

4 Saarland University, Saarland Informatics Campus, Saarbrücken, Germany

5 Univ Lyon, Inria, INSA Lyon, CITI, F-69621 Villeurbanne, France public is engaged in this new-raising space exploration era, inspiring more and more people to pursue space-related careers. This revolution is also affecting missions beyond Earth-orbit based on nano-satellites. Leveraging Commercial Off-The-Shelf (COTS) components, cost-efficient spacecraft have been tested in interplanetary distances [4] and have proven valuable in missions to Mars [5], and others are scheduled for launch to the Moon [6]. Indeed, the new space trend is lowering the cost of access to deep-space, which indicates the realization of a Space Internet that bridges ground, near-Earth, and deep-space assets is imminent in this context.

The establishment of a stable and timely communication link to transport large amount of data to ground can define the success criteria of a mission. Typically, the requirements are aimed at guaranteeing a minimal set of uplink and downlink communication windows. However, to enhance the costeffectiveness, the planning of communications should keep into account different combinations of data volume, relay satellites, and Ground Stations (GSs). This requirement suggests a parametric study of different mission architectures for each lunar site of interest is needed.

In this paper, an algorithm is presented to parametrize and automate the evaluation of the approach, modeling the 
space and ground segment and the possible underlying data handling techniques implemented throughout the mission. In particular, Bent-Pipes (BPs), Store-and-Forwards (S\&Fs), Store Carry-and-Forwards (SC\&Fs) are included in the framework. The algorithm is formulated in such a way that it can be applied to any Lunar network with minimal effort, just by updating the input parameters. This contribution is evaluated and applied to the lava tube case study to determine which of the existing data handling techniques shall be selected for varying mission parameters.

In the study presented in this paper, we consider a lava tube exploration missions on Marius Hills (MH) skylight. The point of interest lays near the equatorial zone, which is of particular scientific interest because it may become the site of a future human settlements [7]. The first inspection of the site should rely on a robotic mission that should map the lava tube surroundings, the skylights, and the inner aspects of the lava tube. A lunar global coverage network has been considered and it is devoted to understanding a possible visible operational plan to sustain the communication on the observation site. The communication architecture of a network of six satellites orbiting the Moon is proposed to assure stable links in the equatorial and near-equatorial regions.

The choice is due to the push toward satellite constellation for future navigation needs and the power generation requirements of the robotic systems. Our algorithm considers the basic communication parameters (antenna gain, frequency, bandwidth) and the coverage analysis to optimize the presented lava tube mission. We claim that this is a representative scenario of most future Lunar missions. In particular, these aspects (multi-hop, navigation/communication dependencies, small and power-constrained satellites, etc.) are common aspects to other Lunar missions on the roadmap. Variants, however, include the exploration of other areas of the Moon, instead of the equatorial area.

The present paper is organized as follows. In Section II, the mission concept, based on the interest in lava tubes, is introduced as a motivation for a Lunar networking architecture presented in Section III, together with an adequate evaluation framework. Evaluation results are presented in Section IV, and Section V summarizes the findings and draws the more important conclusions.

\section{Background}

\subsection{Lunar communications}

Existing Lunar communications are currently handled via ad-hoc and closed (non-interoperable) protocols. In most cases, these are of the bent-pipe type, with possibly data storage as an intermediate step. However, as more Lunar missions are added to the roadmap, the effort of defining a common protocol architecture becomes more relevant.

The Lunar Communications Architecture Working Group from the Interagency Operations Advisory Group (IOAG) has conducted a study for defining a future Lunar Communications Architecture that will facilitate potential cross-support to Lunar missions. These findings are summarized in The Future Lunar Communications Architecture document [8]. The architecture applies to communication assets owned and/or operated by public agencies and their affiliated companies in the private sectors. The overall goal of this report is to achieve an unified position on key technical subjects to ensure a coherent Lunar communications architecture. To this end, 28 Lunar missions comprising a total of 48 space vehicles and involving 10 space agencies during the 20182028 era are evaluated (19 missions would require interoperability services with other missions). Thus, this document clearly depicts the most relevant topics around future Lunar networking protocols and technologies.

In particular, the architecture defined by LCAWG considers: (i) Lunar science and exploration orbiters, (ii) Lunar surface mobile and stationary vehicles, (iii) Lunar relay orbiters, (iv) Earth-orbiting relays that provide service to lunar systems, (v) Lunar Ascent and Descent modules, and (vi) associated Earth ground stations and mission operations centers. Communications links covered by the resulting architecture comprises: (1) Earth-Moon link, (2) Lunar proximity link, (3) Lunar cross link, (4) Lunar surface vicinity link, (5) Earth orbiting relay link, and (6) Earth-space link extension. The physical layer for each of these is defined for both RF and optical communications. Table 1 summarizes the frequency, modulation, coding and link layer protocol considered by the IOAG while compiling their report.

Most relevant missions addressed by the IOAG report are led by NASA. In particular, NASA is developing an extensible and scalable lunar communications and navigation architecture, known as LunaNet [9]. The approach is to move away from the traditional link-centric view to a network-centric concept of operations, where self-contained data units are transported through the system. Due to the delay and disruptions of Lunar links, the popular Internet Protocol (IP) suite nowadays connecting the Internet cannot be directly applied [10]. Instead, NASA is putting forward the Delay/Disruption Tolerant Networking (DTN) protocol stack. DTN core Bundle Protocol specifies the structure of self-contained data units known as bundles and transport rules that include store-and-forward functions to ensure generalized, reliable, and robust internetworking among users and nodes. It is by means of the DTN approach that data via future Lunar networks will likely be handled with storecarry-and-forward approaches. 
Table 1 Lunar communication links characteristics

\begin{tabular}{lllll}
\hline Link & Frequency & Modulation & Coding & Link \\
\hline Earth to Moon & $2025-2110 \mathrm{MHz}$ & PCM/PM/bi-phase-L; & BCH & TC \\
& $7190-7235 \mathrm{MHz}$ & PCM/PM/BPSK PM/PSK/NRZ & LDPC & AOS \\
Moon to Earth & $2200-2290 \mathrm{MHz}$ & PCM/PSK/PM & $\begin{array}{c}\text { Concatenated } \\
\text { (Conv. + RS); }\end{array}$ & TM \\
& & & Turbo; & AOS \\
& $8450-8500 \mathrm{MHz}$ & BPSK & LDPC & \\
& $25.5-27.0 \mathrm{GHz}$ & QPSK; GMSK & Convolutional & \\
$\begin{array}{c}\text { Proximity-Link } \\
\text { (towards lunar } \\
\text { surface) }\end{array}$ & $390-405 \mathrm{MHz}$ & OCM/PM/bi-phase-L & Convolutional & Prox-1 \\
$\begin{array}{c}\text { Proximity-Link } \\
\text { (away from } \\
\text { lunar surface) }\end{array}$ & $435-450 \mathrm{MHz}$ & PCM/PM/bi-phase-L & & \\
\hline
\end{tabular}

Fig. 1 Lava tubes (Marius Hills, left; Mare Ingenii, center; Mare Tranquillitatis, right). Image from SELENE and LRO missions [14]
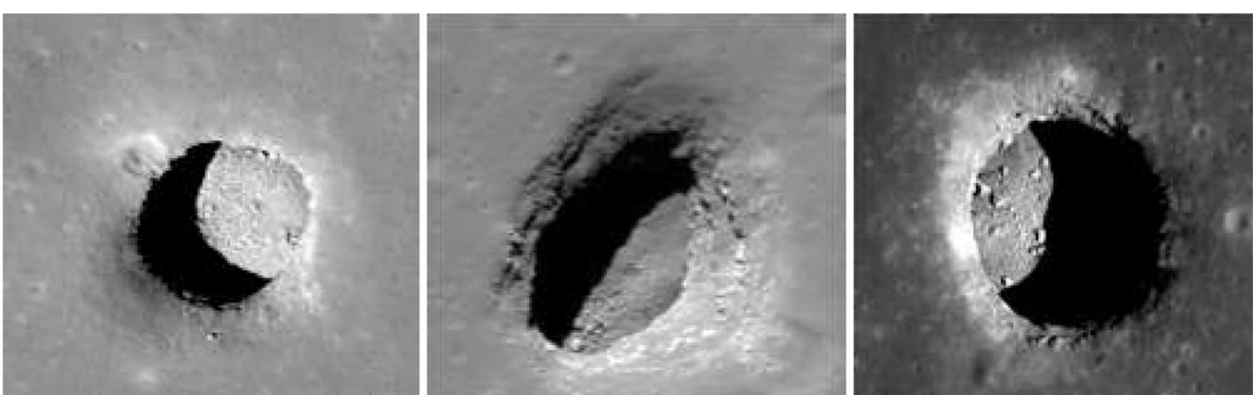

\subsection{Lava tube exploration mission concept}

Lunar lava tubes have raised great interest in the last decade, especially after recent missions such as SELENE and the Lunar Reconnaissance Orbiter (LRO) transmitted highresolution images of these geological formations in the near and the far side of the Moon [11, 12]. Essentially, the lava tubes are volcanic architectures that spread below the surface of the Moon (see Fig. 1).

The main scientific interest focus on mainly three skylights on Moon surface: one in Marius Hills, one in Mare Tranquillitatis and the one in Mare Ingenii, which is in the far side of the Moon [13].

A collection of different mission concepts can be found in NASA's advanced concept reports on lava tubes exploration [14] and in the ESA call Sysnova studies ${ }^{1}$. These missions cover a wide spectrum of possibilities for robotic exploration. There are analyses for landing vehicles inside the lava tubes, spherical robots that can fall and roll in the lava tubes, among other innovative and challenging approaches [15]. However, all of them acknowledges a great uncertainty

\footnotetext{
1 https://www.esa.int/Enabling_Support/Preparing_for_the_Future/ Discovery_and_Preparation/ESA_plans_mission_to_explore_lunar_ caves.
}

regarding the internal structure and geology of the lava tubes, which remains unknown as of today (existing data from LRO and SELENE missions are unfortunately not enough).

The Politecnico di Torino and the ISAE-SUPAERO are studying a robotic mission for the Marius Hills exploration $[16,17]$. The pit in Marius Hills should have a depth and a diameter around fifty meters [13], which facilitates possible accesses to the lava tube. Moreover, there is great interest in the analysis of the terrain of the Marius Hills caves because of its high concentration of iron and titanium-rich minerals [13].

In the case study of this paper, the proposed robotic exploration mission has a payload that generates a considerable quantity of images and radar data to create a 3D map of the lava tube. Therefore, the supporting Lunar satellite network should sustain an efficient transmission of significant data volume from the Moon surface to Earth. Due to the high communication constraints inside the lava tubes, the explorations systems should rely on autonomous decision making and operations. Therefore, self-adaptability without human intervention are of essence for both the exploration's bots and the communication satellites.

The following sections presents an algorithm to evaluate the performance of such autonomous mission frameworks under different networking architectures. 
Table 2 Satellites' parameters: semimajor axis $a$, eccentricity $e$, inclination $i$, true anomaly $v_{0}$, RAAN $\Omega$ and argument of perigee $\omega$

\begin{tabular}{lllllll}
\hline & $S_{1,0}$ & $S_{1,90}$ & $S_{2,0}$ & $S_{2,90}$ & $S_{3,0}$ & $S_{3,90}$ \\
\hline a $(\mathrm{km})$ & 6500 & 6500 & 6500 & 6500 & 6500 & 6500 \\
e $(-)$ & 0.05 & 0.05 & 0.05 & 0.05 & 0.05 & 0.05 \\
i $\left(^{\circ}\right)$ & 40 & 40 & 40 & 40 & 40 & 40 \\
$\nu_{0}\left({ }^{\circ}\right)$ & 0 & 0 & 120 & 120 & 240 & 240 \\
$\Omega\left({ }^{\circ}\right)$ & 0 & 90 & 0 & 90 & 0 & 90 \\
$\omega\left(\left(^{\circ}\right)\right.$ & 90 & 270 & 90 & 270 & 90 & 270 \\
\hline
\end{tabular}

\section{Networking architecture}

The objective of the study is to analyze the communication architecture for a mission in the equatorial region of the Moon, based on the lava tube exploration concept. Usually, the analysis of a communication architecture of a complex scenario like a lunar mission starts with different possibilities. For example, one of the main design drivers to choose the need or not of relay satellites is the power onboard the exploration system and which antenna can be used. After deciding the most suitable general architecture, two processes are optimized in parallel: (1) coverage analysis based on the antenna used, (2) decision on which frequency and bandwidth use for a given amount of telemetry and payload data. Our algorithm is an original contribution that can harmonize and decide upon these last two steps. To this end, we combine the coverage analysis with the other communication parameters to find the fittest architecture in one quick run. Moreover, the proposed approach is scalable and adaptable depending on the specific mission. It can deal with any number of ground stations and with any number of satellites, exploring the best communication configuration depending on the mission requirements. Although it produces coherent results with expectations and previous missions, the data are still rough estimations to be refined in more detailed and specific analysis afterwards. However, the output already helps the designers shape their mission architecture and start planning the operations for future exploration systems. The resulting set-up should achieve a global coverage scenario where at least one satellite is in Line-of-Sight (LOS) per Earth day with the site of interest during the overall mission evaluation period. This will create redundancy in the communications relays: if a satellite fails or enters into safe mode, another satellite can replace it, operating on its behalf. Moreover, at least one communication window per day is required in order to maximize the access toward Earth of the lava tubes' exploration systems, which in turn, will maximize the possible scientific return of the mission.

\subsection{Orbital and ground segment configuration}

To comply with the aforementioned principles, the space segment of the satellite constellation is based on the Lunar global coverage network from [18]. In particular, the constellation is constituted by six satellites around the Moon with a semi-major axis of $6500 \mathrm{~km}$. Three of them have a Right Ascension of the Ascending Node (RAAN) $\Omega=0^{\circ}$, an inclination $i=40^{\circ}$ and different mean anomalies $v$. The remaining three satellites have similar characteristics but have a Right Ascension of the Ascending Node (RAAN) equal to $90^{\circ}$. The orbital parameters and satellites' characteristics are summarized in Table 2.

The ground segment, on the other hand, is comprised of Deep Space Network (DSN) ground stations [19], in particular the ones in California (Goldstone), Australia (Canberra) and Spain (Madrid). The geographic coordinates, with respect to the Earth's or Moon's Latitude and Longitude, are presented in Table 3 .

To evaluate how the communication changes during the various orbits of the Moon around the Earth, the communications opportunities are analyzed for a year long. During the year, the overall elements of the mission have been modeled using the mission analysis tool System Tool Kit (STK), from AGI company [20]. Nonetheless, any tool able to compute accesses between a satellite and a point of interest may be used to create the inputs for the explained simulation framework.

\subsection{Channel and data handling}

Following the guidelines in [21] and looking at previous reference missions [22-24], the payload data communication band was set to X-Band. The telemetry data employs S-Band [23]. The X-Band has relatively few constraints in terms of maximum bandwidth, and it is one of the most used bands for space missions around or on the Moon and Mars. Moreover, it is less susceptible to Earth weather variation like the $\mathrm{K}$ or $\mathrm{Ku}$ band, that are even less restricted in

Table 3 GSs (Earth) and Marius Hills (Moon) geographic coordinates

\begin{tabular}{lllll}
\hline & Canberra & Goldstone & Madrid & Marius Hills \\
\hline La $\left(^{\circ}\right)$ & -35.28 & 35.43 & 40.42 & 13.90 \\
Lo $\left(^{\circ}\right)$ & 149.13 & -116.89 & -4.24 & -56.90 \\
\hline
\end{tabular}


bandwidth. The data rate was set to $150 \mathrm{Mbit} / \mathrm{s}$, following the ITU suggestions [21]. Nevertheless, the overall analysis is parametric and different values of data rate can be used to plan the communications between Earth and Moon using the proposed approach.

Data volumes have been estimated considering the huge number of imaging material from the scientific payload, which should be sent to Earth. Following the approach in [25], a preliminary data volume of $160 \mathrm{~Gb}$ per single transfer was defined for the proposed mission. This value is just a parameter in the simulation: changing the daily data volume is possible to appreciate how the communication schedule can change, and this modification is easily implementable in the proposed model. Indeed, the data volume sensitivity evaluation can be quite interesting during the preliminary phase of the design of a mission, in the domain of conceptual design. This volume of data will be then compressed at a ratio 15:1, following the suggestions of [26].

The possible envisioned data handling approaches are: (1) simple BPs communication, (2) S\&Fs, (3) SC\&Fs architecture. The BP communication relays on the creation of a physical bridge between the lunar site and the DSN network. In this communication mode, the satellite should be in LOS of both the scientific target and the Earth. In the S\&F architecture, the data should be all received within a communication window and should be all sent in another communication window. In the SC\&F architecture, the fraction of data not sent toward Earth from the satellites are stored, and the next useful communication window is used to forward them to Earth. The SC\&F is following the new philosophy of the Delay Tolerant Networks (DTNs) to facilitate and automate communication planning in space-related networks.

\subsection{Evaluation framework}

After defining the physical layer architecture, the satellite constellation and the position of the site of interest, the study continues analyzing the connectivity among Earth, the satellites and the lunar site of interest. Subsequently, all accesses between Marius Hills, each satellite, and each ground station, have been incorporated into a single algorithm for postprocessing. Figure 2 presents a simplified flowchart of the proposed evaluation algorithm, while Algorithm 1 shows a minimum logical working example.

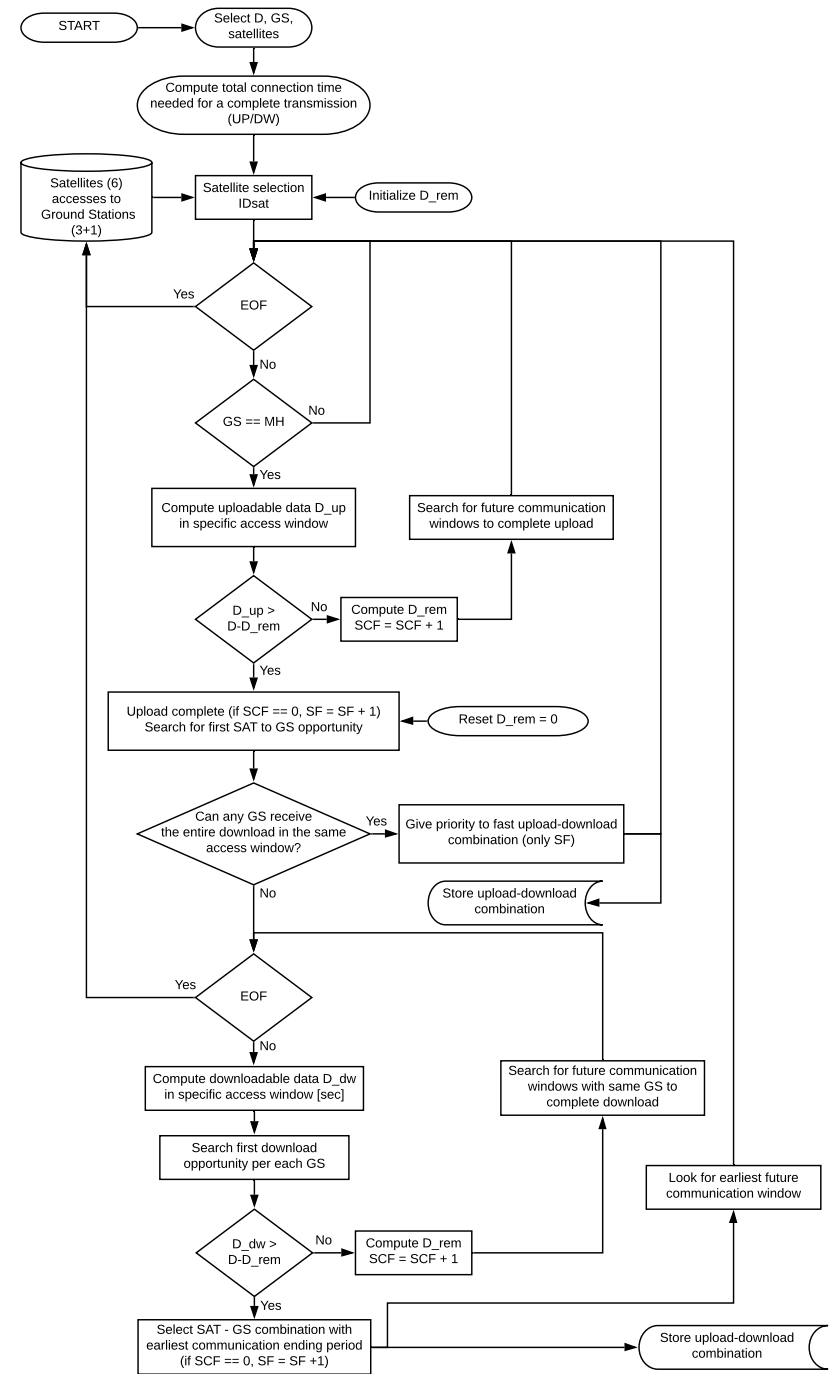

Fig. 2 Algorithm flowchart

The procedure requires in input: the accesses among each satellite and GSs, the chosen data volume $D$ (set at $160 \mathrm{~Gb}$ for this analysis), and the selection of the coupling elements, i.e. the whole set of possibilities and combination of satellites and GSs,. Once these parameters are set, the algorithm combines all accesses in a single file sorted by the starting time. 


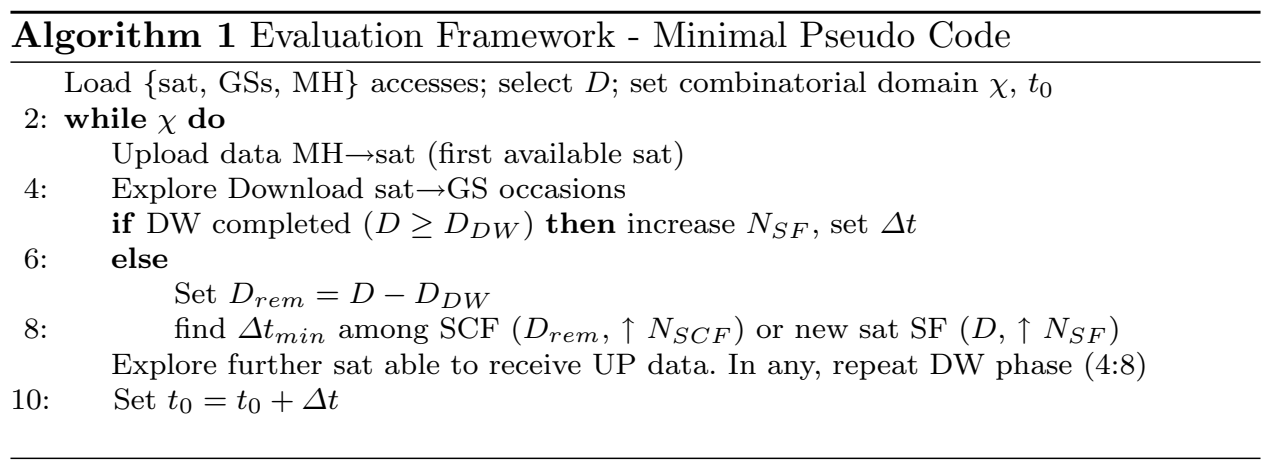

In the first stage, possibilities to upload data from Marius Hills to the first available satellite are considered. Once the upload is completed, that given satellite is excluded from subsequent uploads, but the others can still receive data. Each satellite that has received the data can perform the download phase, by looking for the first subsequent GSs available. The process repeats with all the possible GSs, if available. Downlink contacts that end first, are prioritized in the algorithm; namely, there is a reward process that favors satellites that are usually faster than others to perform the whole upload and download process.

More specifically, both the upload and the download phases can be completed either in a single access window or several. Depending on the chosen $D$, the same access window may be sufficiently large or not to allow a complete transmission, given $t_{0, i}$ and $t_{f, i}$, with $i=1, n$, where $n$ is the total number of available accesses over the whole year. The generic transmission requires a total time $T=f\left(D, t_{d e l}, S F\right)$, where the delay time $t_{d e l}$ considers 32 bits used for the interpacket time, both at the beginning and at the end of the transmission. The single packet size is here considered equal to $S P S=6400$ bits, and a Safety Factor $S F=20 \mathrm{~s}$ is added to the computed minimum transmission time to account for a safe link establishment between transmitter and receiver.

Therefore, $T$ is a value that remains constant once the initial parameters are selected (i.e., all transmissions, both in upload and download, require the same total time). Therefore, a complete transmission can take place if $t_{0, i}+T<t_{f, i}$. In this case, the assumed communication architecture is $\mathrm{S} \& \mathrm{~F}$. .

If the transmission cannot be completed in a single time window, the e SC\&F architecture is still possible and the algorithm calculates the remaining data volume $D_{\text {rem }}$ to then proceed to search for the following opportunities to complete the download phase. It should be noted that the further evaluated contacts assume the same combination of satelliteGround Station (both upload and download) to verify if it is more convenient to proceed with a e SC\&F or to select a new satellite later in time; this evaluation terminates once $D_{\text {rem }}=0$. Total occurrences of the e S\&F and e SC\&F architecture utilization are accumulated for the whole evaluation year for each satellite/Ground Station pair. It is assumed that the first satellite to complete the upload can proceed with the immediate download, and the first Ground Station to receive the entire download is the chosen one among those in range.

The access times are always analyzed by increasing the initial time. However, before proceeding with future access windows, the algorithm first checks if the satellite that completes an upload phase can perform a download in the remaining upload window. This is the only exception that allows a single bi-directional exploration and that does not violate any physical law. This particular operation requires further explanation and, to this end, we present Algorithm 2. 
Fig. 3 Spurious accesses removal

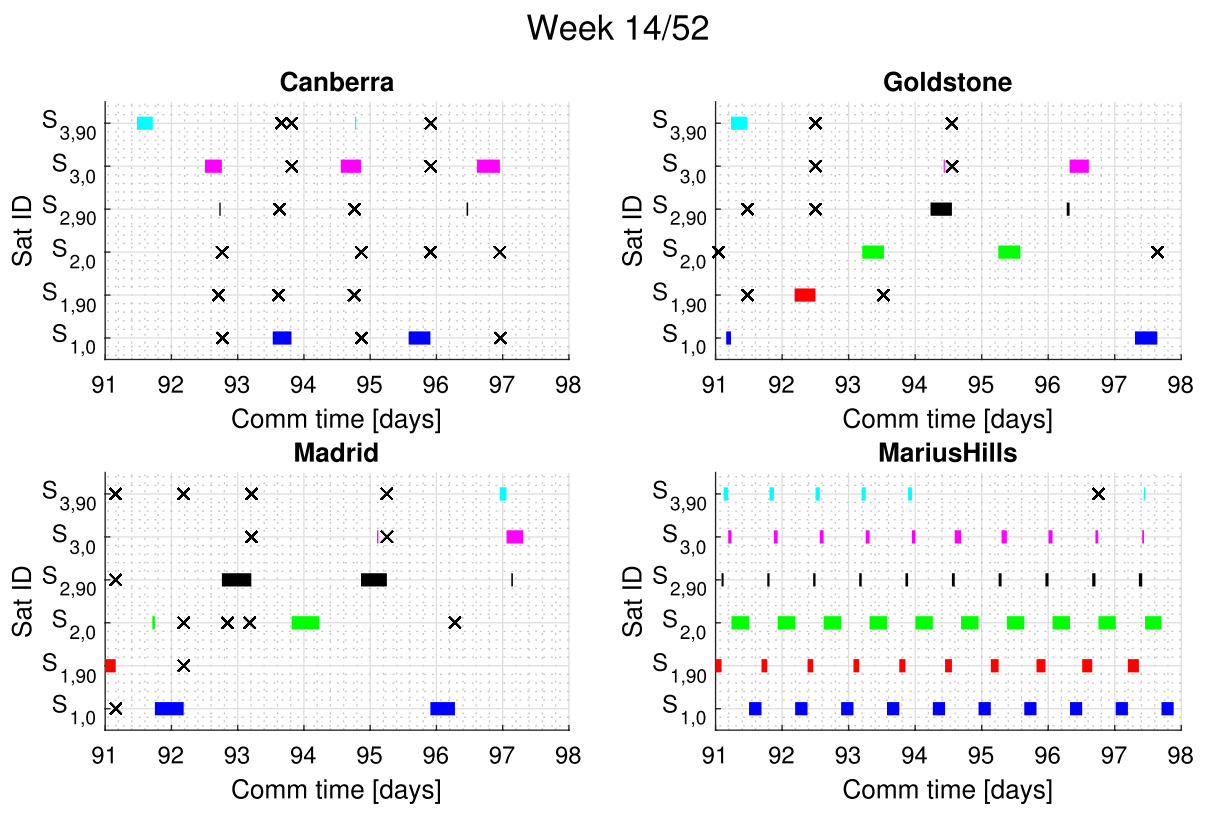

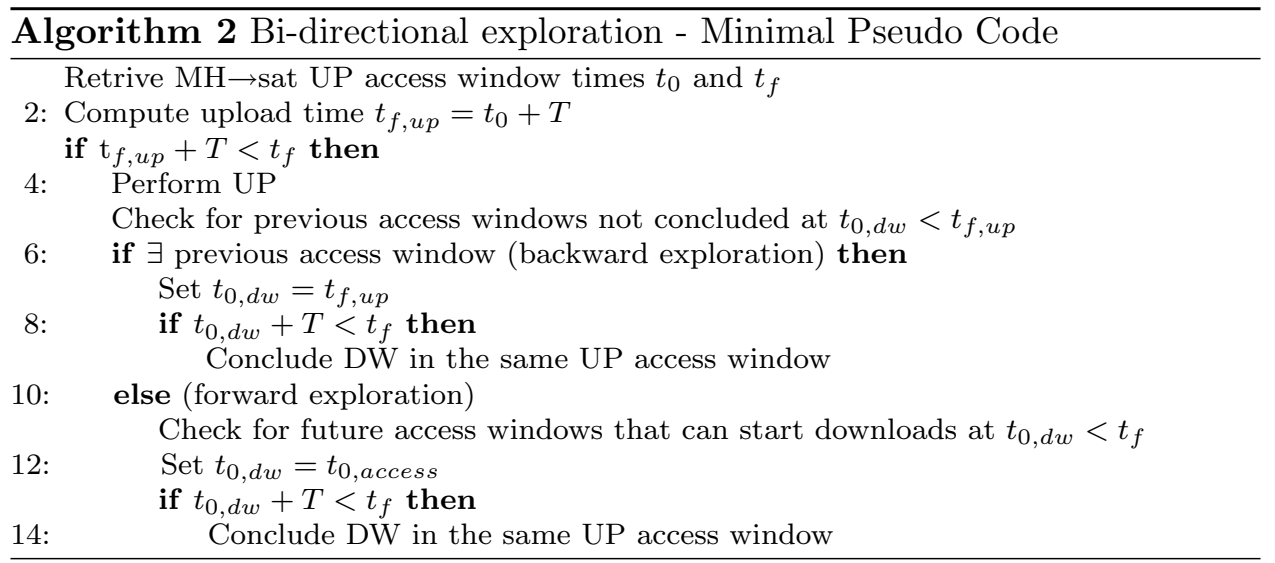

Let $t_{f, u p}=t_{0, i}+T$ be the moment at which the upload phase ends for a specific satellite in the i-th access. The algorithm first checks if there is enough time left $\left(t_{f, u p}+T<t_{f, i}\right)$ in the same window, which spans between $t_{f, u p}$ and $t_{f, i}$. If this applies, it searches through previous accesses started before $\left(t_{0, d w}<t_{f, u p}\right)$ or after $\left(t_{0, d w}>t_{f, u p}\right)$ the upload completion but still not concluded. Once identified the moment $t_{0, d w}$ at which the download can start, if $t_{f, d w}=t_{0, d w}+T<t_{f, i}$, then the same satellite can perform the download phase immediately after the upload without seeking subsequent accesses. This may happen in the best case at $t_{0, d w}=t_{f, u p}$, i.e. as soon as the upload is completed if there is a previous active downlink access window; otherwise in a later moment.

Regardless of this particular bi-directional search, the specific satellite with the uploaded data start to search in all future accesses the occasions to perform the download exploiting a greedy search algorithm. To this end, the satellite considers the type of e S\&F or e SC\&F architecture it uses. Once this happens, the satellite is re-inserted in the list of eligible candidates for the upload, and the cycle begins again.

\section{Simulation results}

Figure 3 shows a post-processing analysis, for a sample week in the considered year (specifically, week 14/52), in which all accesses with a duration less than a certain safety threshold $f\left(t_{d e l}, S F\right)$ are deleted.

Satellites are numbered as $S_{i, \Omega}, i=1,2,3$, and each $S_{i}$ represents a pair of satellites, for a total of 6 satellites in two orbital families, with different RAAN, $\Omega=\{0, \pi / 2\}$. The four sub-images show individual accesses, day by day, for each satellite and for each Ground Station. The deleted 
Fig. 4 Bent pipe architecture-1 month analysis-Marius Hills $\rightarrow$ sat $\rightarrow$ GS

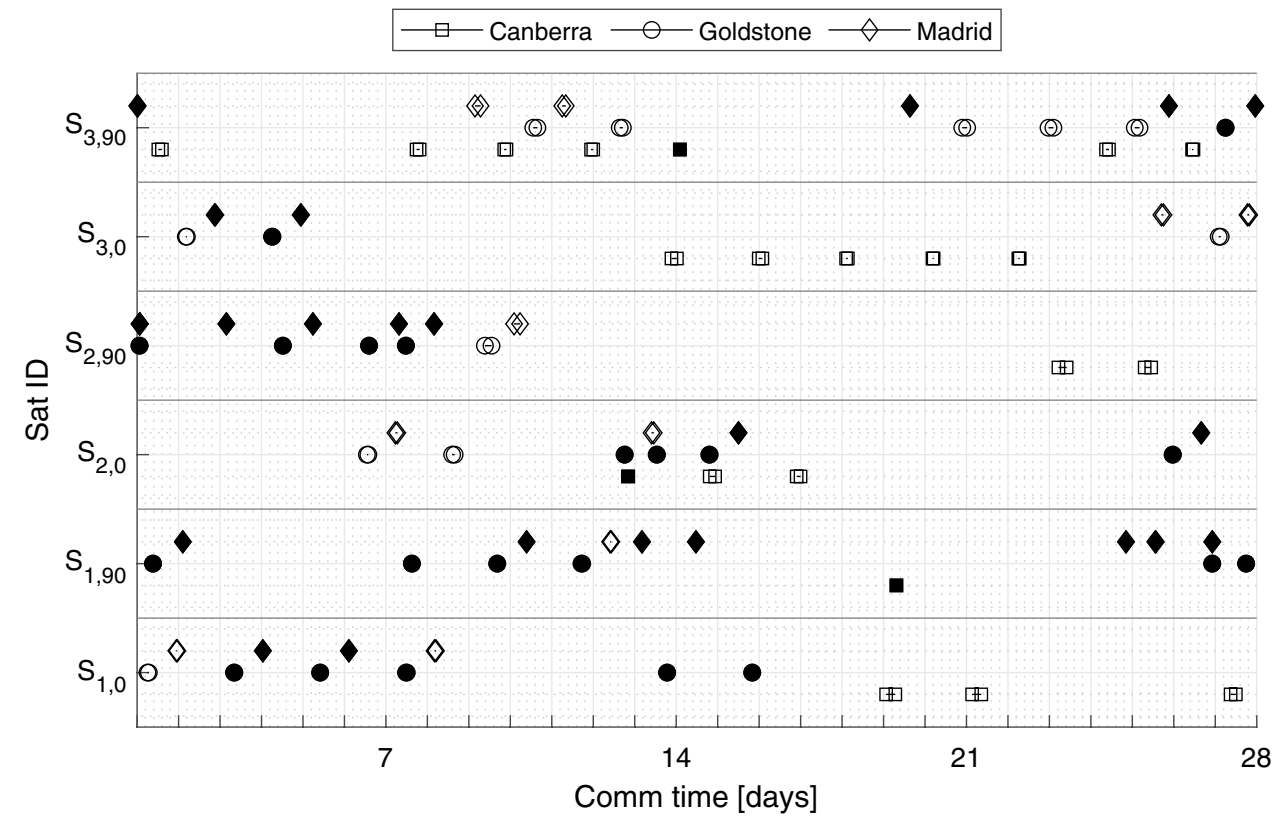

$\nabla \mathrm{S}_{1} \Omega=0 \multimap \mathrm{S}_{1} \Omega=\pi / 2 \multimap \mathrm{S}_{2} \Omega=0 \multimap \mathrm{S}_{2} \Omega=\pi / 2 \multimap \mathrm{S}_{3} \Omega=0 \multimap \mathrm{S}_{3} \Omega=\pi / 2$
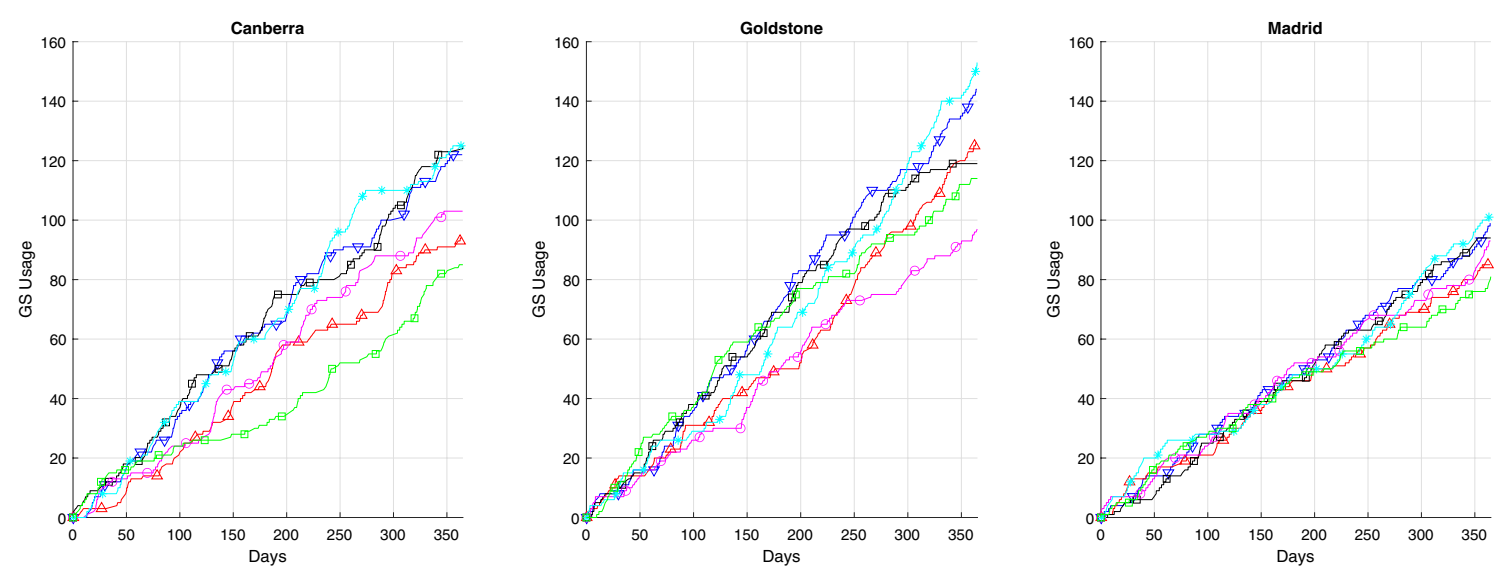

Fig. 5 Separate GS usage from all satellites over the whole year $-D=160 \mathrm{Gbit}$

opportunities (again, those with $\Delta t$ below the safety threshold) are identified with an $\mathrm{x}$ and crossed out. This operation is performed for all the weeks and for all combinations of satellites and GSs, to remove small contact opportunities which will not be considered due to their short duration. Clearly, Marius Hills shows way more access compared to GSs. It is worth pointing out that this post-processing operation does not affect the obtained metrics since such short windows would have been discarded anyway by the evaluation algorithm, but it does increase the computational efficiency.

The first analysis performed is related to the BP architecture. In this case, the satellite must be simultaneously in LOS with both Marius Hills and at least a single Ground
Station. The analysis is performed for the entire year, but in Fig. 4 it is possible to appreciate an excerpt of 28 days in which are shown the trends of the accesses towards Marius Hills and towards each Ground Station for each satellite

Specifically, each satellite level, identified by the name $S_{i, \Omega}$, shows the successful BP communications between a specific Ground Station and Marius Hills through the satellite for a sample month. Two adjacent connected symbols represent the beginning and the end of a specific communication opportunity, while a filled symbol is used for communication windows lasting less that $30 \mathrm{~min}$.

The performance shown repeats qualitatively throughout the year: the BP solution can cover less than $10 \%$ of the available accesses and only for a limited period. Moreover, 

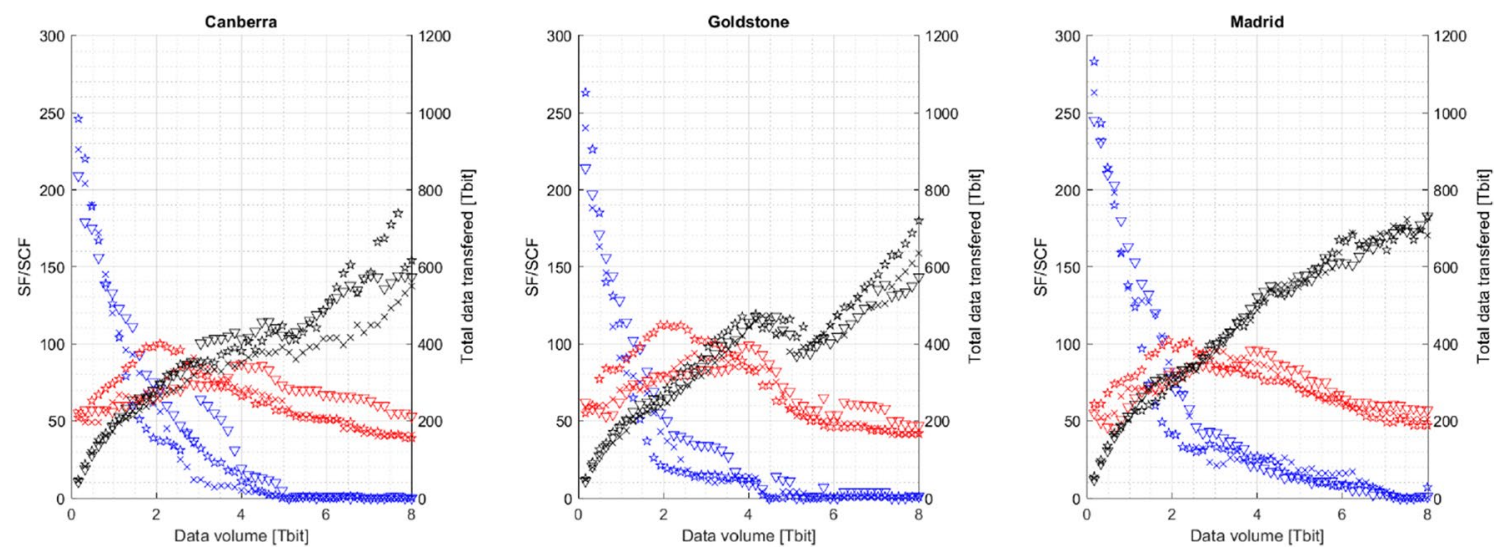

Fig. 6 Year round SF (blue) and SCF (red) architecture usage; total data transferred (black) vs selected data volume; satellites $\left(S_{1} \times, S_{2} \nabla, S_{3} \star\right)$ to separate GSs

for sufficiently large windows (e.g., $6 \mathrm{~h}$ ), even if the communication can take place entirely, it would be unlikely that new data could be produced in the same time window to be sent from Marius Hills to a Ground Station through the satellite via BP. Therefore, the volume of data transferred per access would be reduced. These considerations are the main disadvantage of the BP architecture found in this evaluation.

Therefore, as mentioned in the algorithm's presentation, the focus of the study is to understand the inflection point, i.e. the best trade-off, between the S\&F and SC\&F communication architecture.

Figure 5 shows the usage trend of each Ground Station by each satellite, namely the number of successful accesses, without considering the transferable data or a specific communication architecture. These results derive from the algorithm set to search for the quickest upload and download occasions. It is noticeable that all the satellites with RAAN
$\Omega=\frac{\pi}{2}$, namely $S_{i, 90}, i=1,2,3$, have worse connectivity than those with RAAN $\Omega=0, S_{i, 0}, i=1,2,3$. It is also interesting to observe that there are more accesses to Canberra and Goldstone, compared to Madrid. Nevertheless, fewer accesses do not necessarily imply worse performances or a minor volume of data transferred, as they may indicate that the majority of transmissions happens in a single SC\&F communication.

The algorithm flexibility allows performing a study with the selection of specific permutations of GSs and satellites. For the considerations about coverage and the actual use of individual GSs or satellites, it has proved particularly useful to observe how the results would change with the elimination of one or more of these elements. Throughout the following analysis, it was decided to use only 3 out of the six satellites, precisely the ones with $\Omega=0$, which showed to be the best ones in terms of accesses and transmitted data,
Table 4 Total data $D$ over 1 year (Tbit), S\&F/SC\&F usage value $(N)$ and $\mathrm{S} \& \mathrm{~F}$ percentage $(\%)$ per each satellite and GSs combination for $D=160 \mathrm{Gbit}$

\begin{tabular}{|c|c|c|c|c|c|c|c|c|}
\hline & & \multicolumn{7}{|c|}{ Canberra/Goldstone/Madrid } \\
\hline & &.$\infty \circ$ & $\circ \bullet \circ$ & ০০• & $\cdots \circ$ & $\bullet$ & $\circ \cdots$ & $\cdots$ \\
\hline \multirow[t]{4}{*}{$D_{t o t}$} & $S_{1}$ & 45.12 & 47.87 & 51.23 & 75.71 & 77.47 & 78.80 & 96.08 \\
\hline & $S_{2}$ & 42.26 & 44.19 & 48.03 & 69.15 & 71.70 & 73.95 & 86.10 \\
\hline & $S_{3}$ & 47.95 & 51.03 & 55.19 & 85.39 & 82.19 & 84.95 & 102.99 \\
\hline & $\Sigma$ & 135.33 & 143.09 & 154.45 & 230.25 & 231.36 & 236.70 & 285.17 \\
\hline \multirow[t]{4}{*}{$N$} & $S_{1}$ & $226 / 56$ & $240 / 59$ & $262 / 57$ & $408 / 65$ & $420 / 64$ & $445 / 47$ & $549 / 51$ \\
\hline & $S_{2}$ & $209 / 55$ & $214 / 62$ & $245 / 55$ & $378 / 54$ & $386 / 62$ & $407 / 55$ & $488 / 50$ \\
\hline & $S_{3}$ & $246 / 52$ & $263 / 55$ & $283 / 61$ & $484 / 48$ & $444 / 68$ & $493 / 37$ & $602 / 40$ \\
\hline & $\Sigma$ & $681 / 163$ & $717 / 176$ & $790 / 173$ & $1270 / 167$ & $1250 / 194$ & $1345 / 139$ & $1639 / 141$ \\
\hline \multirow[t]{4}{*}{$\%$} & $S_{1}$ & 80.14 & 80.27 & 82.19 & 86.26 & 86.78 & 90.45 & 91.50 \\
\hline & $S_{2}$ & 79.17 & 77.54 & 81.67 & 87.50 & 86.16 & 88.10 & 90.71 \\
\hline & $S_{3}$ & 82.55 & 82.70 & 82.27 & 90.98 & 86.72 & 93.02 & 93.77 \\
\hline & $\Sigma$ & 80.62 & 80.17 & 82.04 & 88.25 & 86.55 & 90.52 & 91.99 \\
\hline
\end{tabular}

Used $\bullet /$ not used $\circ \mathrm{GSs}$ 
Table 5 Total data $D$ over 1 year (Tbit), S\&F/SC\&F usage (\%) per each satellite and GSs combination for $D=2.08$ Tbit value $(N)$ and $\mathrm{S} \& \mathrm{~F}$ percentage

\begin{tabular}{|c|c|c|c|c|c|c|c|c|}
\hline & & \multicolumn{7}{|c|}{ Canberra/Goldstone/Madrid } \\
\hline & & $\bullet \circ ০$ & $\circ \bullet \circ$ & ০• & $\bullet \circ$ & $\bullet \circ$ & $\circ \bullet \bullet$ & $\cdots$ \\
\hline \multirow[t]{4}{*}{$D_{t o t}$} & $S_{1}$ & 264.54 & 246.86 & 318.32 & 427.02 & 478.22 & 404.90 & 564.54 \\
\hline & $S_{2}$ & 295.16 & 258.62 & 317.23 & 455.97 & 501.33 & 446.68 & 615.73 \\
\hline & $S_{3}$ & 296.32 & 283.94 & 298.51 & 421.02 & 432.28 & 374.27 & 518.32 \\
\hline & $\Sigma$ & 856.02 & 789.42 & 934.06 & 1304.01 & 1411.83 & 1225.85 & 1698.59 \\
\hline \multirow[t]{4}{*}{$N$} & $S_{1}$ & $56 / 71$ & $37 / 80$ & $79 / 74$ & $96 / 109$ & $126 / 105$ & $106 / 89$ & $157 / 116$ \\
\hline & $S_{2}$ & $71 / 66$ & $50 / 77$ & $70 / 82$ & $125 / 90$ & $130 / 103$ & $121 / 95$ & $187 / 102$ \\
\hline & $S_{3}$ & $37 / 100$ & $19 / 112$ & $41 / 97$ & $57 / 140$ & $62 / 140$ & $49 / 124$ & $90 / 153$ \\
\hline & $\Sigma$ & $164 / 237$ & $199 / 269$ & $190 / 253$ & $278 / 339$ & $318 / 348$ & $276 / 308$ & $434 / 371$ \\
\hline \multirow[t]{4}{*}{$\%$} & $S_{1}$ & 44.09 & 31.62 & 51.63 & 46.83 & 54.55 & 54.36 & 57.51 \\
\hline & $S_{2}$ & 51.82 & 39.37 & 46.05 & 58.14 & 55.79 & 56.02 & 64.71 \\
\hline & $S_{3}$ & 27.01 & 14.50 & 29.71 & 28.93 & 30.69 & 28.32 & 37.04 \\
\hline & $\Sigma$ & 40.97 & 28.50 & 42.46 & 44.63 & 47.01 & 46.23 & 53.09 \\
\hline
\end{tabular}

Table 6 Total data $D$ over 1 year (Tbit), S\&F/SC\&F usage value $(N)$ and $\mathrm{S} \& \mathrm{~F}$ percentage (\%) per each satellite and GSs combination for $D=4.00$ Tbit

\begin{tabular}{|c|c|c|c|c|c|c|c|c|}
\hline & & \multicolumn{7}{|c|}{ Canberra/Goldstone/Madrid } \\
\hline & &.$\circ \circ$ & $\circ \bullet \circ$ & ০০• & $\bullet \circ$ & $\bullet$ & $\circ \cdot \bullet$ & $\bullet \cdot$ \\
\hline \multirow[t]{4}{*}{$D_{\text {tot }}$} & $S_{1}$ & 350.04 & 444.25 & 487.15 & 580.87 & 579.48 & 538.75 & 639.37 \\
\hline & $S_{2}$ & 411.94 & 454.98 & 521.32 & 650.22 & 679.13 & 574.37 & 712.89 \\
\hline & $S_{3}$ & 381.05 & 447.99 & 502.47 & 590.25 & 630.90 & 601.69 & 687.92 \\
\hline & $\Sigma$ & 1143.03 & 1347.22 & 1510.94 & 1821.34 & 1889.51 & 1714.81 & 2040.18 \\
\hline \multirow[t]{4}{*}{$N$} & $S_{1}$ & $5 / 70$ & $14 / 85$ & $24 / 87$ & $20 / 113$ & $23 / 110$ & $24 / 99$ & $30 / 117$ \\
\hline & $S_{2}$ & $19 / 87$ & 9/99 & 17.95 & $40 / 126$ & $50 / 123$ & $20 / 113$ & $59 / 127$ \\
\hline & $S_{3}$ & $11 / 66$ & $12 / 83$ & 25.23 & $22 / 105$ & $35 / 100$ & $33 / 94$ & $43 / 108$ \\
\hline & $\Sigma$ & $35 / 223$ & $35 / 267$ & $72 / 263$ & $82 / 344$ & $108 / 333$ & $77 / 306$ & $132 / 352$ \\
\hline \multirow[t]{4}{*}{$\%$} & $S_{1}$ & 6.67 & 14.14 & 21.62 & 15.04 & 17.29 & 19.51 & 20.41 \\
\hline & $S_{2}$ & 17.92 & 8.33 & 17.95 & 24.10 & 28.90 & 15.04 & 31.72 \\
\hline & $S_{3}$ & 14.29 & 12.63 & 25.23 & 17.32 & 25.93 & 25.98 & 28.48 \\
\hline & $\Sigma$ & 12.95 & 11.70 & 21.60 & 18.82 & 24.04 & 20.18 & 26.87 \\
\hline
\end{tabular}

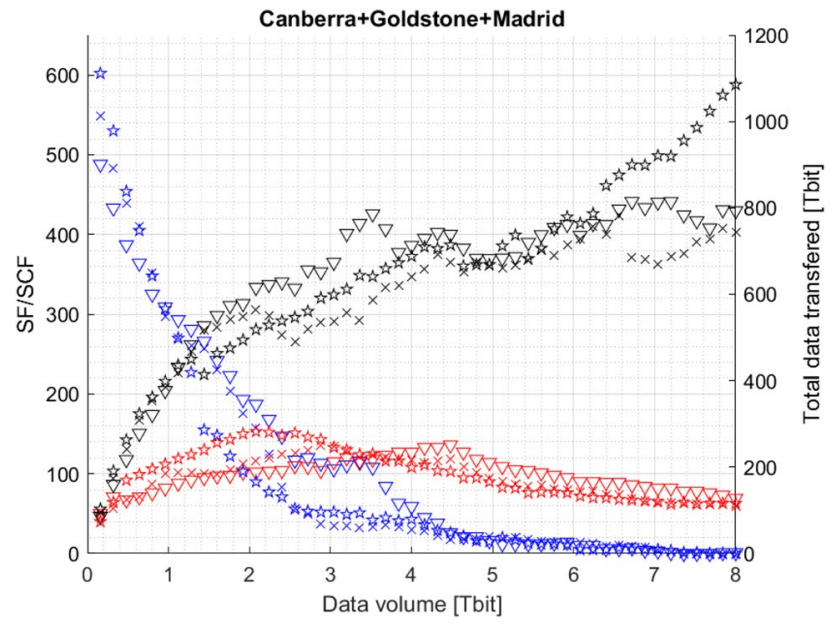

Fig. 7 Year round S\&F (blue) and SC\&F (red) architecture usage; total data transferred (black) vs selected data volume; satellites $\left(S_{1} \times\right.$, $\left.S_{2} \nabla, S_{3} \star\right)$ to aggragated GSs as seen in Fig. 5. Henceforth, they are referred merely as $S_{i}$, $i=1,2,3$.

As can be seen in the following graphs, the analysis was performed under varying combinations of GSs and depending on the desired data volume $D$. Specifically, $D$ ranges between $160 \mathrm{Gbit}$ and 8 Tbit to analyze a broad spectrum of possible or future mission scenarios.

In Fig. 6 the three selected satellites can communicate only with a singular Ground Station, while Fig. 7 shows the scenario in which the algorithm allows a combined use of the most convenient Ground Station time to time.

Figure 6 contains the trends of the combined use of S\&F and SC\&F architectures and the volume of data transferred over the whole year. Both trends are indeed dependent on the data volume $D$. Results show that the use of the S\&F architecture is recommended up to a specific date volume, beyond which the SC\&F architectures prevails. The bifurcation point is around 1.5 Tbit for this scenario and tends to increase its value with the addition of GSs $(\approx 2$ Tbit for 2 
combined GSs, $\approx 2.5$ Tbit for 3 simultaneous GSs, as seen in Fig. 7). In general, this cutting point could oscillate for similar analysis as a function of the orbital parameters of the constellations of satellites, the number of satellites, and the GSs combination. For particularly high $D$, the access windows become too short to allow complete communications and the SC\&F architecture dominates completely.

Figure 7 clearly shows that the combined use of all GSs produce an overall greater data transfer for every data volume selection, while still presenting the mentioned inflection point at a slightly higher threshold, i.e. $D \approx 2.5$ Tbit.

More considerations and further hybrid cases are included in Tables 4, 5 and 6 in the next page, which present the results for three distinct data volume values, i.e. $D=[0.16,2.08,4.00]$ Tbit. In these three examples the three satellites $S_{i}, i=1,2,3$ are communicating for a whole year with one or more GSs. For each scenario, results include the values of the total data volume transferred (single $S_{i}$ and cumulative $\Sigma$ ), the number of accesses in S\&F or SC\&F and the percentage of $\mathrm{S} \& \mathrm{~F}$ use.

Table 4 shows the $D=160$ Gbit case, in which there is a strong prevalence of $\mathrm{S} \& \mathrm{~F}$ architecture. The communication windows are sufficiently large to safeguard almost always a complete transmission. With increasing available GSs also increases the percentage of $S \& F$, as potentially one previously forced SC\&F manages to be covered by an alternative GS. Also increases the overall data transmitted, given that time periods without communications are reduced. It is noteworthy that having eliminated the three satellites $S_{i, 90}$, $i=1,2,3$, has allowed to prefer the use of Madrid, in opposition to the preliminary results noted in Fig. 5: there are higher transmitted data, also traced by the greater number of SF communications. The combination Goldstone-Madrid appears the best between the pairs and is slightly improved in the aggregate scenario.

Table 5 contains the same results with $D=2.08$ Tbit, value beyond the estimated cutting point for the choice of the communication architecture. In fact, the percentage values of S\&F use are almost all below 50\%, showing that for the higher $D$ values the SC\&F architecture is more convenient. Nevertheless, it proves once again that the access windows are still large enough to allow a great part of the communications taking place in a single transmission. The data volume, compared to the previous one, is 10 times higher, but the overall transmitted data values are increased to a lesser extent.

For comparison, Table 6 shows the opposite limit scenario. A data volume $D$ equal to 4 Tbit cannot increase significantly the $D_{t o t}$. The satellites require almost every time the whole available access window to complete a transmission. This consideration comply with both the low values of $\mathrm{S} \& \mathrm{~F} \%$ and with the graphs shown in Fig. 5.

\section{Conclusions}

The cislunar space is on the spot. Lava tubes are among a large set of potentially interesting resources on the Moon that motivates robotic and human exploration in the upcoming years. However, the proper assessment of a supporting Lunar network infrastructures remains an open research topic.

In this paper, we offered a generic algorithm to validate different potential mission architectures that involve a point of interest, a satellite constellation and some GSs on Earth. The whole algorithm is widely adaptable to different scenarios by changing the sole coordinates, orbital parameters, or dispositions of the considered elements. Specifically, we performed a lava tube case study by means of an adequate Lunar network which required non-trivial evaluation to determine the best trade-off between different data handling approaches. Moreover, the algorithm easily accommodates specific needs. For example, if an important action must happen in a specific time frame, e.g. for commanding/ operations, it can be taken into account by imposing that other upload/download operations cannot use that specific opportunity window. This opportunity removal operation is an action that the code already does when discarding, for example, access windows below a certain duration threshold. A good improvement would be to include a GUI to help the user select the ground stations, the accessibility windows, and the satellites; *.mex files and inter-language codes may also be written to let the code work directly with STK, perform the accessibility study, and then perform the communication part described in the paper.

The study outlines an analysis framework for studying and comparing different communication architectures. It uses the mentioned input parameters to provide different possible coupling of GSs and satellites to optimize the amount of data forwarded towards Earth. Therefore, it is possible to schedule the different operations for the robotic exploration systems with respect at the communication opportunities and their duration. Thus, we contributed with an algorithm that ensures a consistent analysis of the case study and any other potential Lunar networks.

Besides extending the study over different Lunar network configurations, future research includes the addition of protocol- and routing-specific techniques into the evaluation loop.

The potential applications of the presented framework are many and adapt to numerous scenarios foreseen or already in development by numerous space agencies. For example, in the framework of the In-Situ Resource Utilization (ISRU), numerous feasibility studies have been completed to demonstrate how specific devices, such as electromagnetic launchers, can be used to transport resources from the lunar soil in 
the vicinity of the Moon [27]; an adequate communications framework can constantly monitor these payloads and may prove to be of invaluable importance, given that a satellite constellation can monitor the payloads' continuous positioning and distribution so to facilitate rendezvous operations. In addition, NASA has recently published a White Paper in which is stated the operative orbit for the future Lunar Orbital Platform-Gateway (LOP-G, also known as DSG) [28]. This particular Near Rectilinear Halo Orbit allows an almost permanent coverage of the Earth's GSs, avoiding eclipses and signal losses: a further interesting application of the presented framework would be to place a satellite constellation on such NRHO, properly spaced apart from the foreseen LOP-G positioning, to further validate what the best configurations and architectures are in this powerful and fundamental scenario and to provide a backup in the rare occasions in which the LOP-G cannot directly communicate with Earth.

Funding Open access funding provided by Politecnico di Torino within the CRUI-CARE Agreement.

Open Access This article is licensed under a Creative Commons Attribution 4.0 International License, which permits use, sharing, adaptation, distribution and reproduction in any medium or format, as long as you give appropriate credit to the original author(s) and the source, provide a link to the Creative Commons licence, and indicate if changes were made. The images or other third party material in this article are included in the article's Creative Commons licence, unless indicated otherwise in a credit line to the material. If material is not included in the article's Creative Commons licence and your intended use is not permitted by statutory regulation or exceeds the permitted use, you will need to obtain permission directly from the copyright holder. To view a copy of this licence, visit http://creativecommons.org/licenses/by/4.0/.

\section{References}

1. National Research Council: The Scientific Context for Exploration of the Moon. The National Academies Press, Washington DC (2007)

2. ISECG: The Global Exploration Roadmap, International Space Exploration Coordination Group (2018)

3. ESA: ESA strategy for science at the Moon, ESA UNCLASSIFIED report (2019)

4. INSPIRE: Interplanetary NanoSpacecraft Pathfinder in Relevant Environment. In: Klesh, A.T., Baker, J. D., Bellardo, J., CastilloRogez, J., Cutler, J., Halatek, L., Lightsey, E.G., Murphy, N., Raymond, C. (eds.) AIAA SPACE 2013 Conference and Exposition (2013)

5. MarCO: Interplanetary Mission Development On a CubeSat Scale Joshua Schoolcraft. In: Klesh, A.T., Werne, T. (eds.) SpaceOps Conference (2016)

6. Bosanac, N., Cox, A.D., Howell, K.C., Folta, D.C.. Trajectory design for a cislunar CubeSat leveraging dynamical systems techniques: The Lunar IceCube Mission (2017)

7. Hufenbach, B., et al.: International missions to lunar vicinity and surface, near-term mission scenario of the global space exploration roadmap. In: 66th International Astronautical Congress, Jerusalem, Israel (2013)
8. Interagency Operations Advisory Group: The Future Lunar Communications Architecture (2019) (final version)

9. Israel, D.J., et al. Lunanet: a flexible and extensible lunar exploration communications and navigation infrastructure. In: 2020 IEEE Aerospace Conference. IEEE (2020)

10. Burleigh, S., Hooke, A., Torgerson, L., Fall, K., Cerf, V., Durst, B., Scott, K., Weiss, H.: Delay-tolerant networking: an approach to interplanetary internet. IEEE Commun. Mag. 41(6), 128-136 (2003)

11. Kaku, T., et al.: Detection of intact lava tubes at MH on the moon by SELENE (Kaguya) lunar radar Sounder. Geophys. Res. Lett. 44(20), 10155-10161 (2017)

12. Lawrence, S.J.: LRO observations of morphology and surface roughness of volcanic cones and lobate lava flows in the MH. J. Geophys. Res. Planets 118(4), 615-634 (2013)

13. Ximenes, S.W., Elliott, J.O., Bannova, O.: Defining a mission architecture and technologies for lunar lava tube reconnaissance. In: 13th ASCE Aerospace Division Conference on Engineering, Science, Construction, and Operations in Challenging Environment (2012)

14. Whittaker, W.: Technologies Enabling Exploration of Skylights, Lava Tubes and Caves, NASA Innovative Advanced Concepts (NIAC) Phase I, Final Report (2012)

15. Whittaker, W.: Exploration of Planetary Skylights and Tunnels, NASA Innovative Advanced Concepts (NIAC) Phase II, Summary Report (2014)

16. Rimani, J., et al.: MBSE Approach Applied to Lunar Surface Exploration Elements, MBSE2020, Noordwijk (2020)

17. Rimani, J., et al.: A Novel Approach to Planetary Rover Guidance, Navigation and Control Based on the Estimation of the Remaining Useful Life, IAC2020, Cyber Edition (2020)

18. Ely, T.A., Lieb, E.: Constellations of elliptical inclined lunar orbits providing polar and global coverage. J. Astron. Sci. 54, 53-67 (2012)

19. NASA, NASA's Mission Operations and Communications Services, NASA report (2009)

20. Systems Tool Kit: STK, Analytical Graphics, Inc. https://www. agi.com/home (2020), accessed 20 July 2021

21. ITU-R Reports: Factors affecting the choice of frequency bands for space research service deep-space telecommunication links, ITU-R Report (2010)

22. LRO (Lunar Reconnaissance Orbiter) + LCROSS. https://direc tory.eoportal.org/web/eoportal/satellite-missions/l/lro (2020), accessed 20 July 2021

23. J.R. Wertz.: Space Mission Engineering: The New SMAD, Microcosm Press, Space Technology Library, vol 28 (2011)

24. IOAG: The Future Lunar Communication Architecture, Report of the Interagency Operations Advisory Group Lunar Communications Architecture Working Group (2019)

25. ESA: MARSFAST ROVER, CDF Study Report (2014)

26. Beser, N.D.: Space Data Compression Standards, Johns Hopkins APL Technical Digest, vol 15 (1994)

27. Mascolo, L., Stoica, A.: Electro-magnetic launchers on the moon: a feasibility study. In: 2018 NASA/ESA Conference on Adaptive Hardware and Systems (AHS), Edinburgh, pp. 37-42 (2018). https://doi.org/10.1109/AHS.2018.8541488

28. National Aeronautics and Space Administration (NASA): White Paper: Gateway Destination Orbit Model: A Continuous 15 Year NRHO Reference Trajectory, August 20, Document ID 20190030294 (2019) 